\title{
QUEEN'S
UNIVERSITY
BELFAST
}

\section{OGLE16aaa - a signature of a hungry supermassive black hole}

Wyrzykowski, Ł., Zieliński, M., Kostrzewa-Rutkowska, Z., Hamanowicz, A., Jonker, P. G., Arcavi, I., Guillochon, J., Brown, P. J., Kozłowski, S., Udalski, A., Szymański, M. K., Soszyński, I., Poleski, R., Pietrukowicz, P., Skowron, J., Mróz, P., Ulaczyk, K., Pawlak, M., Rybicki, K. A., ... Smith, K. (2017). OGLE16aaa - a signature of a hungry supermassive black hole. Monthly Notices of the Royal Astronomical Society: Letters, 465(1), L114L118. https://doi.org/10.1093/mnrasl/slw213

Published in:

Monthly Notices of the Royal Astronomical Society: Letters

\section{Document Version:}

Publisher's PDF, also known as Version of record

\section{Queen's University Belfast - Research Portal:}

Link to publication record in Queen's University Belfast Research Portal

\section{Publisher rights}

(C) 2016 The Authors. This article has been accepted for publication in Monthly Notices of the Royal Astronomical Society: Letters Published by Oxford University Press on behalf of the Royal Astronomical Society. All rights reserved.

\begin{abstract}
General rights
Copyright for the publications made accessible via the Queen's University Belfast Research Portal is retained by the author(s) and / or other copyright owners and it is a condition of accessing these publications that users recognise and abide by the legal requirements associated
\end{abstract} with these rights.

Take down policy

The Research Portal is Queen's institutional repository that provides access to Queen's research output. Every effort has been made to ensure that content in the Research Portal does not infringe any person's rights, or applicable UK laws. If you discover content in the Research Portal that you believe breaches copyright or violates any law, please contact openaccess@qub.ac.uk. 


\title{
OGLE16aaa - a signature of a hungry supermassive black hole
}

\author{
Łukasz Wyrzykowski, ${ }^{1 \star}$ M. Zieliński, ${ }^{1}$ Z. Kostrzewa-Rutkowska, ${ }^{1,2,3}$ \\ A. Hamanowicz, ${ }^{1}$ P. G. Jonker, ${ }^{2,3 \star}$ I. Arcavi, ${ }^{4,5}$ J. Guillochon, ${ }^{6}$ P. J. Brown, ${ }^{7}$ \\ S. Kozłowski, ${ }^{1}$ A. Udalski, ${ }^{1}$ M. K. Szymański, ${ }^{1}$ I. Soszyński, ${ }^{1}$ R. Poleski, ${ }^{1,8}$ \\ P. Pietrukowicz, ${ }^{1}$ J. Skowron, ${ }^{1}$ P. Mróz, ${ }^{1}$ K. Ulaczyk, ${ }^{1,9}$ M. Pawlak, ${ }^{1}$ K. A. Rybicki, ${ }^{1}$ \\ J. Greiner, ${ }^{10}$ T. Krühler, ${ }^{10}$ J. Bolmer, ${ }^{10,11}$ S. J. Smartt, ${ }^{12}$ K. Maguire ${ }^{12}$ and K. Smith ${ }^{12}$ \\ ${ }^{1}$ Warsaw University Astronomical Observatory, Al. Ujazdowskie 4, PL-00-478 Warszawa, Poland \\ ${ }^{2} S R O N$, Netherlands Institute for Space Research, Sorbonnelaan 2, NL-3584 CA Utrecht, the Netherlands \\ ${ }^{3}$ Department of Astrophysics/IMAPP, Radboud University Nijmegen, PO Box 9010, NL-6500 GL Nijmegen, the Netherlands \\ ${ }^{4}$ Las Cumbres Observatory Global Telescope Network, 6740 Cortona Dr Ste 102, Goleta, CA 93117-5575, USA \\ ${ }^{5}$ Kavli Institute for Theoretical Physics, University of California, Santa Barbara, CA 93106-4030, USA \\ ${ }^{6}$ Harvard-Smithsonian Center for Astrophysics, 60 Garden St, Cambridge, MA 02138, USA \\ ${ }^{7}$ George P. and Cynthia Woods Mitchell Institute for Fundamental Physics and Astronomy, Texas A \& M University, \\ Department of Physics and Astronomy, 4242 TAMU, College Station, TX 77843, USA \\ ${ }^{8}$ Ohio State University, Department of Astronomy, 140 West 18th Avenue, Columbus, OH 43210, USA \\ ${ }^{9}$ Department of Physics, University of Warwick, Gibbet Hill Road, Coventry CV4 7AL, UK \\ ${ }^{10}$ Max-Planck Institute for Extraterrestrial Physics, D-85748 Garching, Giessenbachstr. 1, Germany \\ ${ }^{11}$ European Southern Observatory, D-85748 Garching, Germany \\ ${ }^{12}$ Astrophysics Research Centre, School of Mathematics and Physics, Queens University Belfast, Belfast BT7 1NN, UK
}

Accepted 2016 October 13. Received 2016 October 11; in original form 2016 June 7; Editorial Decision 2016 October 11

\begin{abstract}
We present the discovery and first three months of follow-up observations of a currently ongoing unusual transient detected by the Optical Gravitational Lensing Experiment (OGLE-IV) survey, located in the centre of a galaxy at redshift $z=0.1655$. The long rise to absolute magnitude of $-20.5 \mathrm{mag}$, slow decline, very broad $\mathrm{He}$ and $\mathrm{H}$ spectral features make OGLE16aaa similar to other optical/UV tidal disruption events (TDEs). Weak narrow emission lines in the spectrum and archival photometric observations suggest the host galaxy is a weak-line active galactic nucleus, which has been accreting at higher rate in the past. OGLE16aaa, along with SDSS J0748, seems to form a sub-class of TDEs by weakly or recently active supermassive black holes (SMBHs). This class might bridge the TDEs by quiescent SMBHs and flares observed as 'changing-look quasars', if we interpret the latter as TDEs. If this picture is true, the previously applied requirement for identifying a flare as a TDE that it had to come from an inactive nucleus, could be leading to observational bias in TDE selection, thus affecting TDE-rate estimations.
\end{abstract}

Key words: black hole physics - galaxies: active-galaxies: individual: OGLE16aaa.

\section{INTRODUCTION}

It has become a paradigm that nearly all galaxies at current times harbour a supermassive black hole (SMBH) in their centre (e.g. Magorrian et al. 1998). In the cold dark matter ( $\lambda$ CDM) theory of cosmology, current (redshift zero) galaxies are the product of hierarchical mergers of smaller galaxies. These smaller building blocks also host black holes in their centres (Kormendy \& Richstone 1995, Greene 2012), potentially intermediate-mass black holes (IMBHs), with masses from 100 to $10000 \mathrm{M}_{\odot}$. After two galaxies merge, the two

*E-mail: 1w@astrouw.edu.pl (LW), name pronunciation: Woocash Vizhikovski; p.jonker@sron.nl (PGJ) black holes will merge as well (see Begelman, Blandford \& Rees 1980). Therefore, mergers of black holes may play an important role in building SMBHs (cf. Pelupessy, Di Matteo \& Ciardi 2007). Interestingly, SMBHs with masses of more than a billion solar mass have been found already at redshifts of more than 6 when the Universe was less than 1 Gyr old (see Fan 2006). Such SMBHs may be seeded by $100 \mathrm{M}_{\odot}$ black holes at redshifts $z>15$, which then grow by uninterrupted accretion of gas at the Eddington rate with a standard radiative efficiency of 10 per cent (e.g. Haiman 2013). However, quasars grow only for about $\approx 4.5 \times 10^{7} \mathrm{yr}$ before feedback stops the gas supply (Silk \& Rees 1998). In order to solve this problem, one can start with more massive black holes such as IMBHs and/or allow mass to be accreted at a rate higher than the 
Eddington limit and/or have part of the black hole growth be due to mergers of black holes.

Tidal disruption events (TDEs, e.g. Hills 1975, Rees 1988), in which a star is torn apart by the tidal forces of the SMBH, offer a unique opportunity to study the mass distribution of SMBHs. The intrinsic TDE rate should be dominated by the SMBHs with the lowest mass (Wang \& Merritt 2004, Stone \& Metzger 2016), so volume-complete TDE samples can measure the occupation fraction of IMBHs in small galactic bulges informing SMBH formation theories. However, the inhomogeneous and small sample of TDEs currently available, found either in X-rays (e.g. Bade, Komossa \& Dahlem 1996, Nikołajuk \& Walter 2013) or in the UV/optical (e.g. van Velzen et al. 2011, Gezari et al. 2012, Arcavi et al. 2014, Holoien et al. 2014) prevents us from discriminating between various emission mechanisms of TDEs and hence from conclusions on the SMBH mass function (e.g. Stone \& Metzger 2016).

Optical/UV TDEs are relatively luminous $\left(M_{\text {peak }} \sim-20\right)$ and blue $\left(T \sim\right.$ few $10^{4} \mathrm{~K}$ ) few-months- to years-long transients with broad $\mathrm{H}$ and/or He II emission lines (Arcavi et al. 2014). Arcavi et al. (2014) and French, Arcavi \& Zabludoff (2016) noted that 75 per cent of optically found TDEs occurred in quiescent Balmer-strong (called $\mathrm{E}+\mathrm{A}$ by some) galaxies, which account for 2.3 per cent of Sloan Digital Sky Survey (SDSS) galaxies. Such galaxies are thought to be products of a recent merger (within $1 \mathrm{Gyr}$ ), which triggered an observed increase in star formation (Balmer absorption series in their spectra are caused by a significant amount of A-type stars). That preference might be due to disturbed dynamics of the nuclear star cluster, or the presence of a coalescing binary black hole, causing nearby stars to go on a collision course with their central black holes (e.g. Wegg \& Nate Bode 2011).

However, a fraction of the optical/UV TDEs seems to not match such scenario. Host-galaxy spectra of extreme coronal line emitters (ECLE), SDSS J095209.56+214313.3 (Komossa 2008; Palaversa 2016) and SDSS J0748 (Wang et al. 2011), as well as ASASSN-14li (Holoien et al. 2016) and PTF09axc (Arcavi et al. 2014) this weak, narrow emission lines, which could be indicative of a weak active galactic nucleus (AGN) present in the core.

AGNs are known to exhibit photometric variability at a level of few tens of magnitude (e.g. Kozłowski et al. 2016). However, occasionally, flares are observed well above the level of their typical variability, both in X-rays (e.g. Strotjohann et al. 2016) and optical (e.g. Tanaka 2013). The reasons for such significant changes in mass accretion rate are still under debate and include binary black hole interactions with the disc as well as stellar disruptions.

Long-term and wide-field spectroscopic and photometric data obtained by the SDSS, as well as on-going transient searches [All Sky Automated Survey for Supernovae (ASAS-SN), Panoramic Survey Telescope \& Rapid Response System (Pan-STARRS), Mobile Astronomy System of Telescope Robots (MASTER) surveys], have revealed nearby AGNs (Seyfert galaxies), which changed their spectral characteristics, often accompanied with a temporal increase in observed flux, e.g. Lawrence et al. (2016). In particular, an event observed in the quasar SDSS J0159+0033 (LaMassa et al. 2015), has been recently interpreted as a TDE by a massive SMBH (Merloni et al. 2015). In large fraction of other similar object, so-called changing-look quasars (hereafter, $\mathrm{CH}-\mathrm{L}-\mathrm{QSO}$ ) (MacLeod et al. 2016), the appearance of a broad component to the $\mathrm{H} \alpha$ emission line was found to be transient (Baldassare et al. 2016), i.e. the broad line disappeared on the time-scale of years. Moreover, one of such CH-L-QSOs, found in NGC 2617 by ASAS-SN in 2013 (Shappee et al. 2014) has recently been reported to show a re-brightening (Oknyansky et al. 2016a). If those flares are indeed due to TDEs, the stellar disruption must be induced fairly frequently, namely, on

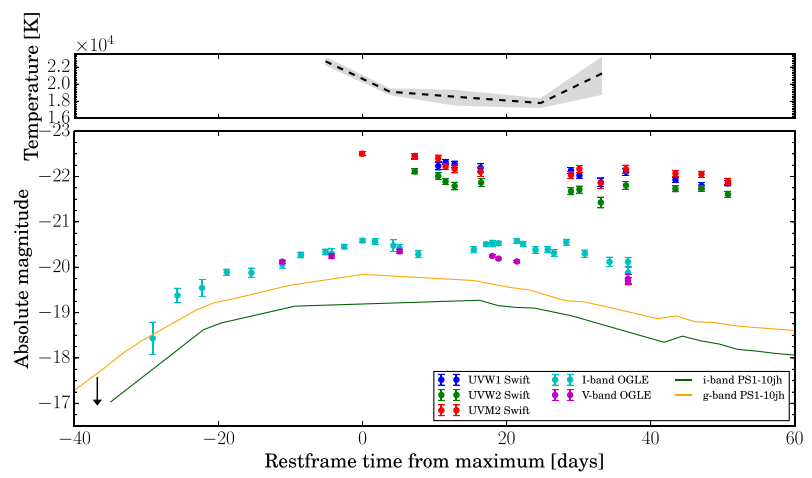

Figure 1. Photometric observations (bottom) and temperature (top) evolution with $1 \sigma$ uncertainty (grey) of the OGLE16aaa transient. Also shown is the light curve of the TDE PS1-10jh (Gezari et al. 2012). An arrow shows the last non-detection in OGLE.
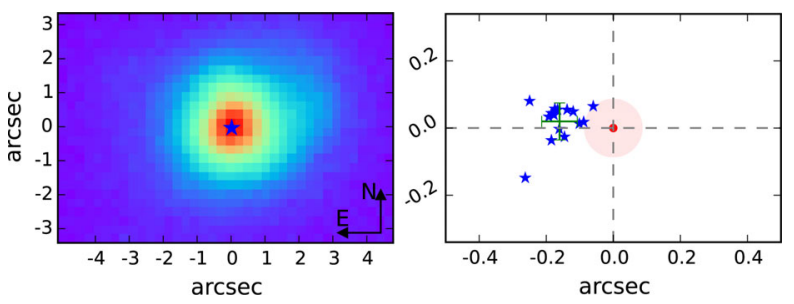

Figure 2. Left: mean position of the OGLE16aaa transient (asterisk) on false colour deep stack of $\sim 100$ pre-flare OGLE $I$-band images. Right: positions of the detections when the source was brightest (blue) with respect to the centre of the galaxy (red circle). The green cross marks the mean position of the transient offset from the centre by $160 \pm 140$ mas, when including the error-bar on the best-fitted galaxy position.

a time-scale of years. An X-ray TDE candidate in an AGN IC 3599 was found to show a re-occurrence period of about nine years (Campana et al. 2015), which could be explained with a binary central black hole.

In this Letter, we describe the discovery and first months of follow-up of an unusual transient, OGLE16aaa. We propose that OGLE16aaa is a TDE candidate in a low-luminosity AGN host galaxy, and it may be another link in the chain of disruption events which occur from quiescent to active galaxy cores.

\section{DISCOVERY AND EARLY FOLLOW-UP}

OGLE16aaa (Fig. 1) was discovered by the OGLE transient detection system (Wyrzykowski et al. 2014) ${ }^{1}$, a programme within the Optical Gravitational Lensing Experiment (OGLE-IV; Udalski, Szymański \& Szymański 2015). This transient, located at RA, Dec. $(\mathrm{J} 2000.0)=1: 07: 20.88,-64: 16: 20.7$, was found during visual inspection of candidates detected by the automated pipeline on 2016 January 2, at $I$-band magnitude $\sim 20$ mag (Wyrzykowski et al. 2016). It was found at the centre of GALEXASC J010720.81-641621.4 galaxy of $17.00 \pm 0.01 \mathrm{mag}$ as measured by OGLE in the $I$ band and $20.83 \pm 0.12$ and $21.82 \pm 0.32 \mathrm{mag}$ in GALEX near-ultraviolet and far-ultraviolet, respectively. Fig. 2 shows the host-galaxy image from a deep stack of $\sim 100$ OGLE $I$-band images (all taken before the event) with the position of the transient.

On 2016 Jan 17, the Public ESO Spectroscopic Survey for Transients $^{2}$ (PESSTO; Smartt et al. 2015) took a spectrum of

\footnotetext{
${ }^{1}$ http://ogle.astrouw.edu.pl/ogle4/transients

${ }^{2}$ www.pessto.org
} 


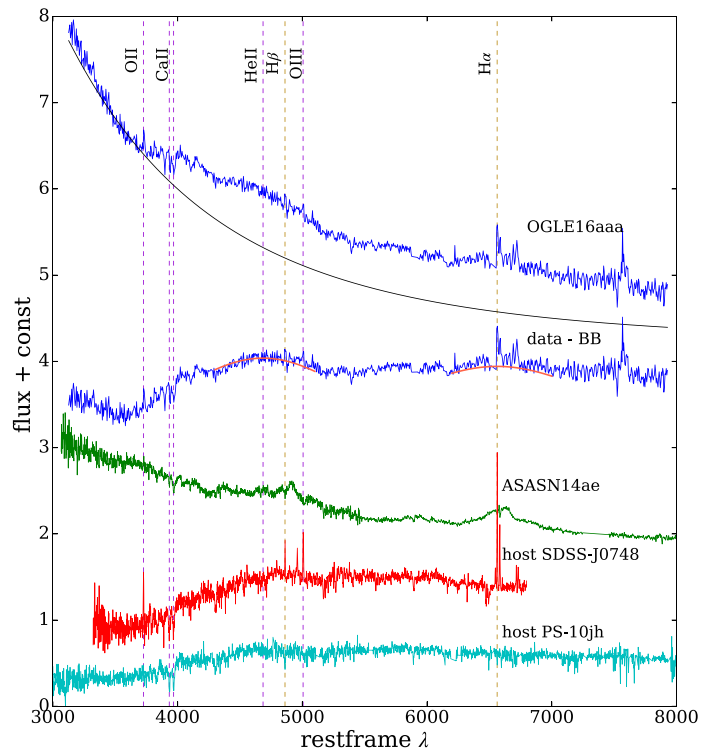

Figure 3. Top: PESSTO spectrum of OGLE16aaa flare (with the Gr\#13 and $1.0 \operatorname{arcsec}$ slit setup) at $-3 \mathrm{~d}$ of the $I$-band maximum. The solid line shows the blackbody model with $T=22000 \mathrm{~K}$ best matching to the blue part of the spectrum. Below is the same spectrum, but with BB model subtracted. The orange Gaussians indicate the location of broad spectra components at $\mathrm{He}$ II and $\mathrm{H} \alpha$. We also show spectra of TDE-candidate hosts with narrow emission lines. Also shown are the spectra of hosts of other TDE candidates: SDSS J0748 and PS-10jh.

OGLE16aaa with the European Southern Observatory (ESO)/New Technology Telescope (NTT) ESO Faint Object Spectrograph and Camera (EFOSC2) instrument (Firth et al. 2016) (top of Fig. 3). The spectrum (which is available on Weizmann Interactive Supernova data REPository (WISEREP), Yaron \& Gal-Yam 2012) shows a blue continuum with broad $\mathrm{He}$ II and $\mathrm{H} \alpha$ and weak emission and absorption lines consistent with a redshift of $z=$ $0.1655(\sim 800 \mathrm{Mpc})$. The light curve reached an $I$-band maximum on 2016-01-20.1 at $18.98 \pm 0.05 \mathrm{mag}$. Assuming standard cosmology $\left(\Omega_{\mathrm{M}}=0.28, H_{0}=70\right)$, and taking into account only the Galactic extinction towards this source of $A_{I}=0.028$ mag (Schlafly \& Finkbeiner 2011), the maximum brightness corresponds to an absolute magnitude of $-20.5 \mathrm{mag}$, in range consistent with previous TDEs (Arcavi et al. 2014).

The transient was then followed-up from the ground with the Gamma-Ray Burst Optical/Near-Infrared Detector (GROND) instrument on the Max Planck Institute for Extraterrestial Physics (MPE) $2.2 \mathrm{~m}$ telescope in La Silla (Greiner et al. 2008) and with Swift satellite's Ultra-Violet Optical Telescope (UVOT) from 201601-19 (Greiner et al. 2016 and Zhang, Yu \& Yan 2016). For details on Swift data reductions see Brown et al. (2014).

Difference imaging photometry conducted by OGLE on the densely sampled pre-discovery images spanning about $3.5 \mathrm{yr}$ showed no prior flaring nor variable activities in the core of this galaxy at a level below 1 per cent. The last non-detection on HJD 2457364.61144 and first detection on 2457373.5795 at $21.1 \pm$ $0.4 \mathrm{mag}$, allow us to constrain the beginning of the event to about HJD $=2457369 \pm 4$ d (see Fig. 1).

\section{HOST AND TRANSIENT CHARACTERISTICS}

Location - the offset of the transient from the galaxy's photocentre, obtained from the OGLE I-band images using Difference Image Analysis (DIA), is less than $160 \pm 140$ mas $(460 \pm 400 \mathrm{pc}$ for $z=0.1655$ ), as shown in Fig. 2, and is consistent with the position of the nucleus.

Host galaxy - from the light distribution of the host on the deep OGLE $I$-band image, we obtained a Sersić index of $n=1.08$, which corresponds to a black hole mass of $\log M_{h}=6.58\left(\approx 4 \times 10^{6} \mathrm{M}_{\odot}\right)$ using the relation in Savorgnan (2016).

Since there is, to our knowledge, no pre-flare host spectrum available, we have to wait until the event fades out completely to obtain the spectrum of the nucleus of the galaxy alone. However, the Le PHARE (Photometric Analysis for Redshift Estimate) (Ilbert et al. 2006) best matching template spectrum to the spectral energy distribution from archival UV, optical, near- and far-infrared observations is found for a galaxy with a stellar mass of $\log M=10.3 \pm 0.2 \mathrm{M}_{\odot}$, with no strong evidence for star formation.

Signatures of the host are already present in the PESSTO spectrum of the flare. The spectrum shows weak [O III], [O II] and [N II] narrow forbidden lines in emission as well as narrow emission of $\mathrm{H} \alpha$ and $\mathrm{H} \beta$. Assuming that the broad features are from the transient, and the narrow ones are from the host, the host spectrum resembles that of SDSS J0748 (Wang et al. 2011), which hosted a TDE candidate. The galaxy does not seem to belong to the quiescent Balmer-strong galaxies, which were noted to host most of the optically discovered TDEs so far (French et al. 2016). The line ratios $(\log ([\mathrm{NII}] / \mathrm{H} \alpha)$ $=-0.43$ versus $\log ([\mathrm{OIII}] / \mathrm{H} \beta)=-0.25)$ indicate there is a mixture of star formation in the galaxy of OGLE16aaa as well as a weak AGN in its core. The line ratios are also similar to those in the host of SDSS J0748. Moreover, the Wide-field Infrared Survey Explorer (WISE) (Wright et al. 2010) colours for OGLE16aaa and SDSS J0748 place those hosts near the region occupied by AGNs, following the classification method of Assef et al. (2010). However, a spectrum taken after the transient emission fades is still required for a quantitative comparison to other TDE hosts and for disentangling the continuum level of the transient from that of the host.

The host galaxy is seen face-on and has no obvious signature of spiral structure, apart from a faint hint of an extended structure to the west from the centre (Fig. 2).

Flare spectrum - the flare spectrum, taken by PESSTO at -3 rest-frame days from the $I$-band maximum, supplemented with the earliest Swift observation in UVM2, taken at around the peak, is consistent with blackbody model with temperature higher than $22000 \mathrm{~K}$. The host emission is clearly present in the spectrum at wavelengths longer than rest-frame $4000 \AA$. The residual spectrum, after subtraction of the blackbody continuum, shows two broad emission features around $\mathrm{He}$ II and $\mathrm{H} \alpha$, as seen in all optically selected TDEs. Arcavi et al. (2014) reported on a continuum of broad $\mathrm{He}$ II and $\mathrm{H} \alpha$ emission lines (after the host-galaxy light has been subtracted) in the spectra of TDEs. OGLE16aaa seems to fit the picture very well, though with somewhat higher velocity dispersion $\left[\sim 23000\right.$ and $\sim 19000 \mathrm{~km} \mathrm{~s}^{-1}$ for $\mathrm{He}$ II and $\mathrm{H} \alpha$ lines, respectively, corresponding to full widths at half-maximum of $\sim 850 \AA\left(54000 \mathrm{~km} \mathrm{~s}^{-1}\right)$ and $\sim 970 \AA\left(45000 \mathrm{~km} \mathrm{~s}^{-1}\right)$, respectively].

Light curve - There is no apparent flaring nor variability activity in the historical (3.5 yr) OGLE $I$-band light curve of the nucleus of the host galaxy at a level below $I \sim 22$ mag (host subtracted), less than 1 per cent of host light. The optical light curve reached the peak in about 30 rest-frame days and then exhibited significant variability, particularly around 15 rest-frame days after $I$-band maximum. Comparing our brightest UV measurements to the archival GALEX data, we estimate the overall UV amplitude of the flare of about 3 mag. The overall decline of the light curve in both optical and UV is very slow; however, the actual slope of the decline is yet to be determined in the observations in the second half of 2016. No $\mathrm{X}$-ray emission was detected by $S$ wift/X-ray Telescope (XRT) at a 


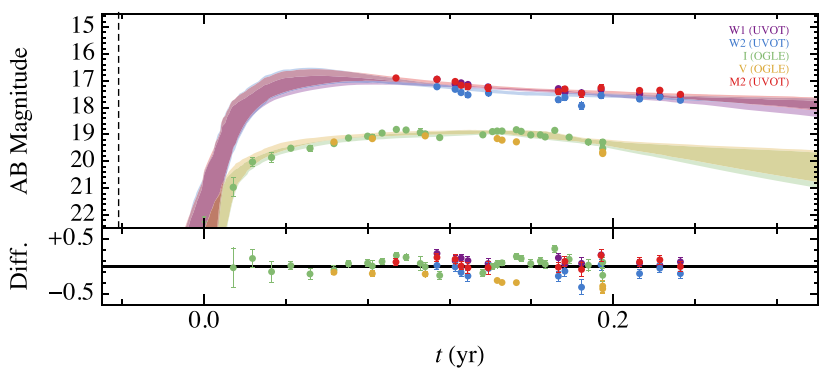

Figure 4. TDEFit model of the OGLE16aaa transient fit to OGLE and Swift data. The shaded regions in the upper panel show the $1 \sigma$ spread in the model fits, whereas the lower panel shows residuals of the highest scoring model.

level above 0.002 counts $\mathrm{s}^{-1}(3 \sigma)$, corresponding to an upper limit for the unabsorbed luminosity of $5 \times 10^{42} \mathrm{erg} \mathrm{s}^{-1}(0.3-10 \mathrm{keV})$, for a power law with photon index of -2 .

Tidal disruption model - we fit OGLE16aaa's photometry with the tidal disruption light-curve fitting software TDEFIT (Guillochon, Manukian \& Ramirez-Ruiz 2014; Vinkó et al. 2015) (see Fig. 4), a Monte Carlo modelling code. For OGLE16aaa, we presume that the observed light comes from a combination of the light produced by a viscously driven disc component (Guillochon \& McCourt 2016), emission from circularization (Jiang, Guillochon \& Loeb 2016), and reprocessing of light from the debris which ensheaths the accretion disc structure (Guillochon et al. 2014). We assume a flat prior for $M_{\mathrm{h}}$ which allows all black hole masses between $10^{4}$ and $10^{9} \mathrm{M}_{\odot}$, a prior on impact parameter $\beta$ which assumes pinhole scattering $(P(\beta) \propto$ $\beta^{-2}$ ), a Kroupa stellar mass function (Kroupa 2001), and flat priors on all other parameters. We find that the most likely combination of disruption parameters is $M_{\mathrm{h}}=10^{6.2 \pm 0.1} \mathrm{M}_{\odot}, M_{*}=10^{-0.5 \pm 0.4} \mathrm{M}_{\odot}$ (between 0.1 and $0.8 \mathrm{M}_{\odot}$, median $\sim 0.3 \mathrm{M}_{\odot}$ ) and $\beta=1.77_{-0.53}^{+0.94}$, with a degeneracy between a sub-solar star suffering a full disruption and a solar star suffering a partial disruption. The total observed energy emitted in the event is about $5 \times 10^{52} \mathrm{erg}$. For assumed (median) 8 per cent of efficiency, the total accreted mass is therefore about $0.3 \mathrm{M}_{\odot}$, indicating either complete or partial disruption of the star and suggesting that the mass of the star was probably higher than $0.6 \mathrm{M}_{\odot}$.

\section{DISCUSSION}

The characteristics of OGLE16aaa resemble those of other optically found TDEs. First, the flare's location coincides with the nucleus of the host galaxy. Also, the derived photospheric blackbody temperature remains high $(\sim 20000 \mathrm{~K})$ throughout the available data for this event, significantly higher than in typical supernovae. Moreover, the temperature seems to rise at 35 rest-frame days from I-band maximum, as seen before in ASASSN-14ae TDE (Holoien et al. 2014). Both the optical light curve and the presence of very broad He II and $\mathrm{H} \alpha$ in the spectra resemble those of other optical/UV TDEs (Arcavi et al. 2014). OGLE16aaa seems, therefore, to be a TDE. Lack of any variability in $3.5 \mathrm{yr}$ prior to the flare and large amplitude of the flare strongly disfavours regular AGN flaring.

However, the underlying host galaxy is somewhat different in OGLE16aaa than in most of known TDEs so far. French et al. (2016) have shown that most optically found TDEs detected so far occur in quiescent, Balmer-strong galaxies, which are considered post-mergers. The Balmer absorption line series are not present in case of OGLE16aaa; however, a deep post-flare spectrum is still needed to verify it. Another TDE candidate, SDSS J0748, also does not show Balmer series and is an outlier on fig. 2 of French et al. (2016).

Among the X-ray-detected TDEs, IGR J12580, interpreted as due to a flare due to disruption of a Jupiter-mass planet was also detected in a weak AGN/Seyfert galaxy NGC4845 (Nikołajuk \& Walter 2013). The narrow emission lines ratios in all those three hosts, as well as their WISE colours indicate that the host contains weak AGN.

For most TDEs found so far, the black hole was assumed dormant, since there was no evidence to the contrary. Here, we propose that OGLE16aaa and several other TDE candidates are due to a stellar disruption in a weak AGN or Seyfert 1-type galaxy, where narrow emission lines originate from the circumnuclear material, photoionized by X-ray photons generated due to accretion. Bennert et al. (2006) showed that for several Seyfert II galaxies the projected distance of the narrow-line region extends to hundreds and even thousands of parsecs. Such accretion is likely to have been due to regular AGN accretion, but it could also have been due to a previous TDE. TDEs are expected to repeat on time-scales of $10^{4} \mathrm{yr}$ (Wang $\&$ Merritt 2004), and if the paths from the lines of sight from the narrow-line region to us represent a broad delay function, we could still see an echo of previous TDEs in the spectra (e.g. Wegg \& Nate Bode 2011).

Moreover, the TDEs in narrow emission line hosts seem to be bridging stellar disruptions by dormant SMBHs and those in much more active AGN, which exhibit a transient appearance of broadline $\mathrm{He}$ II and/or $\mathrm{H} \alpha$ and increase in blue continuum in previously QSO-like spectra with strong narrow emission lines (e.g. MacLeod et al. 2016). Merloni et al. (2015) has already suggested that the first example of CH-L-QSO from Stripe 82 can actually be a stellar disruption on a fairly massive $\mathrm{SMBH}\left(\sim 10^{8} \mathrm{M}_{\odot}\right)$. In such a case, the observed effect of a star getting too close to the main engine is the luminosity increase due to an increase in accretion rate (blue continuum from the thermal emission) and the disrupted material allows for the formation of broad $\mathrm{He}$ II and $\mathrm{H} \alpha$ lines with very large dispersion. If TDEs are common enough, especially in postmerger galaxies, where the disruption rate is increased either due to dynamical instabilities of the nuclear cluster, or presence of a binary black hole, they could play an important role in growth of nuclear black holes (e.g. Hills 1975, Volonteri, Sikora \& Lasota 2007).

While AGN are known to vary on small scales, none of the hosts of weak AGN TDEs exhibit any significant pre-flare photometric variability. Primarily, this is due to lack of long-term photometric data for most of them. OGLE16aaa is probably the first TDE candidate in a weak AGN where we have a long (3.5 yr) history of pre-flare observations indicating no detectable variability.

Based on pre-flare variability, van Velzen et al. (2011) rejected several candidates in Stripe 82 data, and since then, all the current surveys are using this criterion when selecting TDEs among nuclear flares. However, as already suggested in Strotjohann et al. (2016), significant and temporal (flare-like) changes in the accretion in AGN are hard to explain with changes in gas flow and can be attributed to disruptions of stars. Restricting nuclear flares solely to apparently quiet nuclei might introduce biases in observational optically selected TDE rate determinations.

The approximate monthly variability observed in the optical and UV bands during the flare and seen in the residuals of the TDE model remains puzzling in OGLE16aaa. Possible explanations include precession of the disc (e.g. Janiuk \& Czerny 2011; Tchekhovskoy 2014), duty cycle imposed by the orbital period of the returning debris (Jiang et al. 2016), or even a binary SMBH (Liu, Li \& 
Komossa 2014). Further observations are required to address this issue.

\section{CONCLUSIONS}

In the course of OGLE-IV search for extragalactic transients, we have discovered a new candidate for a TDE of a $0.1-0.8 \mathrm{M}_{\odot}$ star by a $10^{6.2} \mathrm{M}_{\odot} \mathrm{SMBH}$.

OGLE16aaa event, along with SDSS J095209.56+214313.3, SDSS J0748, ASASSN-14li and IGR J1258 seem to form a subclass of TDEs in galaxies hosting a weak AGN, with weak narrow emission lines. Moreover, the existence of such TDEs supports one of the explanations for so-called CH-L-QSOs, where persistent strong narrow emission lines get superimposed with variable broad emission lines.

A possible explanation of the observed variability in the light curve of OGLE16aaa is that it is induced by a binary black hole on a tight orbit or due to disc precession or circularization on a timescale of about a month; however, further multimessenger follow-up is required to understand this.

The fact that TDEs could also be found in (weak) AGNs is important for determining TDE rates, since currently there is likely an observational bias against selection of optically found TDEs. Whereas only 10 per cent of galaxies host an AGN, this bias could be larger given that AGN activity is triggered or enhanced by recent mergers and milliparsec-scale binary SMBHs could strongly enhance the TDE rate.

\section{ACKNOWLEDGEMENTS}

We would like to thank Drs Marek Nikołajuk, Dan Maoz, Jozsef Vinko and Sjoert van Velzen, and the attendees of the Warsaw Black Hole Lunches, for helpful discussions. OGLE Team thanks Prof.. M. Kubiak and G. Pietrzyński.

$€ W, A H$ and KR were supported by Polish National Science Centre grant OPUS 2015/17/B/ST9/03167. PGJ and ZKR acknowledge support from European Research Council (ERC) Consolidator Grant 647208. OGLE project has received funding from the Polish NCN, grant MAESTRO 2014/14/A/ST9/00121 to AU. IA acknowledges the Karp Discovery Award. This work is based (in part) on ESO PESSTO programme 188.D-3003, 191.D-0935. SJS acknowledges ERC grant 291222 and Science \& Technology Facilities Council (STFC) grants ST/I001123/1 and ST/L000709/1. We acknowledge the use of NED data base (ned.ipac.caltech.edu) and TDE catalogue (tde.space).

\section{REFERENCES}

Arcavi I. et al., 2014, ApJ, 793, 38

Assef R. J. et al., 2010, ApJ, 713, 970

Bade N., Komossa S., Dahlem M., 1996, A\&A, 309, L35

Baldassare V. F. et al., 2016, ApJ, 829, 57

Begelman M. C., Blandford R. D., Rees M. J., 1980, Nature, 287, 307

Bennert N., Jungwiert B., Komossa S., Haas M., Chini R., 2006, A\&A, 459, 55

Brown P. J., Breeveld A. A., Holland S., Kuin P., Pritchard T., 2014, Ap\&SS, 354,89

Campana S., Mainetti D., Colpi M., Lodato G., D’Avanzo P., Evans P. A., Moretti A., 2015, A\&A, 581, A17

Fan X., 2006, New Astron. Rev., 50, 665

Firth R. et al., 2016, Astron. Telegram, 8559, 1

French K. D., Arcavi I., Zabludoff A., 2016, ApJ, 818, L21

Gehrels N. et al., 2004, ApJ, 611, 1005

Gezari S. et al., 2012, Nature, 485, 217
Greene J. E., 2012, Nature Commun., 3, 1304

Greiner J. et al., 2008, PASP, 120, 405

Greiner J. et al., 2016, Astron. Telegram, 8579, 1

Guillochon J., Manukian H., Ramirez-Ruiz E., 2014, ApJ, 783, 23

Guillochon J., McCourt M., 2016, ApJL, preprint (arXiv:1609.08160)

Haiman Z., 2013, Astrophysics and Space Science Library, Vol. 396, The First Galaxies. Springer-Verlag, Berlin, p. 293

Hills J. G., 1975, Nature, 254, 295

Holoien T. W.-S. et al., 2014, MNRAS, 445, 3263

Holoien T. W.-S. et al., 2016, MNRAS, 455, 2918

Ilbert O. et al., 2006, A\&A, 457, 841

Janiuk A., Czerny B., 2011, MNRAS, 414, 2186

Jiang Y., Guillochon J., Loeb A., 2016, ApJ, 830, 125

Komossa S. et al., 2008, ApJ, 678, L13

Kormendy J., Richstone D., 1995, ARA\&A, 33, 581

Kozłowski S., 2016, ApJ, 826, 118

Kroupa P., 2001, MNRAS, 322, 231

LaMassa S. M. et al., 2015, ApJ, 800, 144

Lawrence A. et al., 2016, MNRAS, 463, 296

Liu F. K., Li S., Komossa S., 2014, ApJ, 786, 103

MacLeod C. L. et al., 2016, MNRAS, 457, 389

Magorrian J. et al., 1998, AJ, 115, 2285

Merloni A. et al., 2015, MNRAS, 452, 69

Nikołajuk M., Walter R., 2013, A\&A, 552, A75

Oknyansky V. L. et al., 2016, Astron. Telegram, 9015, 1

Palaversa L., Gezari S., Sesar B., Stuart J. S., Wozniak P., Holl B., Ivezić Ž., 2016, ApJ, 819, 151

Pelupessy F. I., Di Matteo T., Ciardi B., 2007, ApJ, 665, 107

Rees M. J., 1988, Nature, 333, 523

Savorgnan G. A. D., 2016, ApJ, 821, 88

Schlafly E. F., Finkbeiner D. P., 2011, ApJ, 737, 103

Shappee B. J. et al., 2014, ApJ, 788, 48

Silk J., Rees M. J., 1998, A\&A, 331, L1

Smartt S. J. et al., 2015, A\&A, 579, A40

Stone N. C., Metzger B. D., 2016, MNRAS, 455, 859

Strotjohann N. L., Saxton R. D., Starling R. L. C., Esquej P., Read A. M., Evans P. A., Miniutti G., 2016, A\&A, 592, A74

Tanaka T. L., 2013, MNRAS, 434, 2275

Tchekhovskoy A., Metzger B. D., Giannios D., Kelley L. Z., 2014, MNRAS, 437, 2744

Udalski A., Szymański M. K., Szymański G., 2015, Acta Astron., 65, 1

van Velzen S. et al., 2011, ApJ, 741, 73

Vinkó J. et al., 2015, ApJ, 798, 12

Volonteri M., Sikora M., Lasota J.-P., 2007, ApJ, 667, 704

Wang J., Merritt D., 2004, ApJ, 600, 149

Wang T.-G., Zhou H.-Y., Wang L.-F., Lu H.-L., Xu D., 2011, ApJ, 740, 85

Wegg C., Nate Bode J., 2011, ApJ, 738, L8

Wright E. L. et al., 2010, AJ, 140, 1868

Wyrzykowski Ł. et al., 2014, Acta Astron., 64, 197

Wyrzykowski L. et al., 2016, Astron. Telegram, 8577, 1

Yaron O., Gal-Yam A., 2012, PASP, 124, 668

Zhang W., Yu W., Yan Z., 2016, Astron. Telegram, 8644, 1

\section{SUPPORTING INFORMATION}

Additional Supporting Information may be found in the online version of this article:

\section{suplementary.tar}

(http://www.mnrasl.oxfordjournals.org/lookup/suppl/doi:10.1093 /mnrasl/slw213/-/DC1).

Please note: Oxford University Press is not responsible for the content or functionality of any supporting materials supplied by the authors. Any queries (other than missing material) should be directed to the corresponding author for the article.

This paper has been typeset from a $\mathrm{T}_{\mathrm{E}} \mathrm{X} / \mathrm{L} \mathrm{T} \mathrm{E} \mathrm{X}$ file prepared by the author. 